\title{
SAGES guidelines for the introduction of new technology and techniques
}

\author{
Herbert P. Lerner
}

Received: 22 April 2014/ Accepted: 26 April 2014/Published online: 27 June 2014

(C) Society of American Gastrointestinal and Endoscopic Surgeons (SAGES) 2014

Drs. Stefanidis, Fanelli, Price, and Richardson, in concert with the SAGES Guidelines Committee, have, in their Guidelines for The Introduction of New Technologies and Techniques, provided a comprehensive and forward-looking approach to the introduction of new technologies and techniques into the practice of medicine and surgery. These Guidelines provide a straightforward approach for physicians to adapt into their practices, and which will provide assurance that technological and technical advancement provides enhanced safety and benefit for patients from their implementation. Together with SAGES and other professional organizations, as well as the FDA, innovators, physicians and patients who continue to search for better outcomes in healthcare will benefit from these guidelines.

Hippocrates, in his Epidemics, states that a physician "must be able to tell the antecedents, know the present, and foretell the future-must mediate these things, and have two special objects in view with regard to disease, namely to do good or to do no harm" [1]. As new techniques and technologies are introduced into the clinical arena, it is important to remember these edicts and to include patients into the decision-making process as we embrace these changes.

Each year at the SAGES Scientific Session, the exhibit hall is filled with the next generation of devices for a variety of disease states, such as GERD, obesity, and endoscopic surgery. As I look back at my training over 30 years ago, it

"No official support or endorsement of this article by the Food and Drug Administration is intended or should be inferred."

H. P. Lerner $(\bowtie)$

US Food and Drug Administration, Silver Spring,

MD 20993, USA

e-mail: herbert.lerner@fda.hhs.gov excites me to see the technological changes that have changed the practice of surgery so dramatically over the years, as well as changes in techniques for performing the procedures with these devices. Oh too well do I remember seeing the surgeon's back as I held a retractor during an open cholecystectomy, and was then asked about the procedure I had just witnessed! However, at what cost are we making these advancements? As outlined in a recent editorial in Surgical Endoscopy, for GERD treatments we may be at a point of conflicting priorities as industry promotes new technologies, surgeons incorporate them into their practices, and the cost of healthcare continues to rise [2].

At the FDA's Center for Devices and Radiological Health, we are tasked with evaluating new technologies and determining whether or not the supporting data demonstrates a reasonable assurance that the devices we review are safe and effective. Ensuring the safety of medical devices for the general public is complicated by the increasing incidence of complications of surgical devices and paucity of uniform criteria and processes, as adroitly noted by the authors. It is well understood by the FDA that the data collected for approval through the pre-market approval (PMA) process may not provide complete information regarding the safety and effectiveness of a device once it becomes available to the general public. Simultaneously, we recognize that new technologies are being developed and studied abroad before being brought to the US market, and we have made a commitment to reverse that trend and work with sponsors to bring their trials to the US so that our patients can benefit from these technologies faster. It is also obvious that changes in technology bring changes in technique. Although the agency does not regulate techniques (i.e., practice of medicine), the authors appropriately and clearly recommend that surgical device techniques be assessed in a manner to protect patients. 
As the authors note, a recent survey of SAGES members indicated a need for guidelines for gastrointestinal and endoscopic surgical devices. Based on a survey of SAGES Board members and their clinical experience and knowledge of the literature, the authors propose seven guidelines for implementing changes in technology and technique into clinical practice, each with an assessment of quality of evidence and strength of recommendation. While the quality of evidence varies among the guidelines, the strength of the recommendations were generally strong. Taken together, these recommendations provide a clear pathway for the introduction of new technologies and techniques into clinical practice that should be recognized by practitioners as a model to follow. Specifics as to the implementation of these new technologies or techniques, including training, mentoring, credentialing, etc., can be varied depending on institutional and/or society requirements, but at the very least there must be minimum guidelines that must be met for each point. In addition, steps must be taken to assure that patients are well informed as new devices or new techniques are introduced into clinical practice.

Conflict of interest None.

\section{References}

1. Of the Epidemics written by Hippocrates in 400BCE. http:// classics.mit.edu/Hippocrates/epidemics.mb.txt

2. Smith CD (2013) SAGES clinical spotlight review: endoluminal treatment for gastroesophageal reflux disease (GERD). Surg Endosc 27:2655-2657 